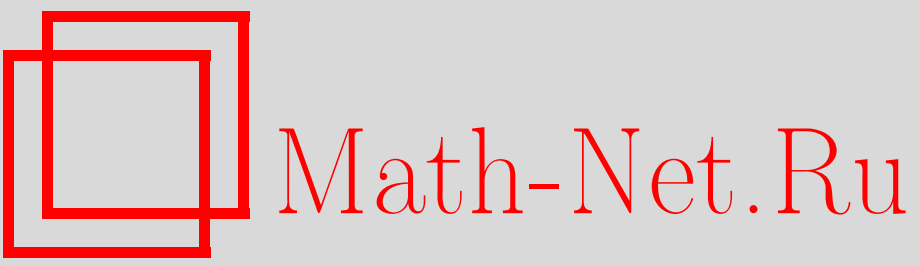

А. И. Кириллов, Стохастическое квантование с помощью модифицированного уравнения Ланжевена, ТМФ, 1998, том 115, номер 1, 46-55

DOI: https://doi.org/10.4213/tmf856

Использование Общероссийского математического портала Math-Net.Ru подразумевает, что вы прочитали и согласны с пользовательским соглашением

http://www.mathnet.ru/rus/agreement

Параметры загрузки:

IP: 54.172 .240 .79

26 апреля 2023 г., 05:02:52 
Том 115, № 1

апрель, 1998

\section{СТОХАСТИЧЕСКОЕ КВАНТОВАНИЕ С ПОМОЩЬЮ МОДИФИЦИРОВАННОГО УРАВНЕНИЯ ЛАНЖЕВЕНА}

Для евклидовой модели квантового поля типа синус-Гордон доказаны существование решений у модифицированного уравнения Ланжевена и сходимость вероятностных распределений этих решений к некоторой мере. Никаких регуляризаций в функционале действия не используется. Размерность пространства-времени произвольна.

\section{ВВЕДЕНИЕ}

В данной работе исследуется квантовое поле, классическая евклидова модель которого определяется следуюшим функционалом действия:

$$
A(f)=\frac{1}{2} \int_{\mathbb{R}^{d}}\left[f(x)^{2}+\sum_{i=1}^{d}\left(\frac{\partial f(x)}{\partial x_{i}}\right)^{2}+v(f(x))\right] d x
$$

здесь $d$ - размерность пространства-времени и $v$ - заданная функция. Она предполагается дифференцируемой и такой, что $\forall z_{1}, z_{2} \in \mathbb{R}$ справедливо неравенство

$$
\left|v^{\prime}\left(z_{1}\right)-v^{\prime}\left(z_{2}\right)\right|<\lambda\left|z_{1}-z_{2}\right| \quad \text { и } \quad\left|v^{\prime}\left(z_{1}\right)\right|<\gamma\left|z_{1}\right|,
$$

где $\lambda$ и $\gamma$ - некоторые положительные числа.

Модели такого типа исследовались в работах $[1,2]$. В данной работе мы продолжаем эти исследования с помошью метода стохастического квантования. Мы вводим вспомогательную переменную $t \in \mathbb{R}_{+}=\left[0, \infty\left[\right.\right.$ и строим случайный процесс $t \in \mathbb{R}_{+} \mapsto \varphi_{t}$ со значениями в пространстве $S^{\prime}\left(\mathbb{R}^{d}\right)$ обобшенных функций умеренного роста такой, что функции Швингера рассматриваемой модели определяются следуюшим равенством:

$$
\mathfrak{S}_{n}\left(x_{1}, \ldots, x_{n}\right)=\lim _{t \rightarrow \infty} \mathrm{E} \varphi_{t}\left(x_{1}\right) \ldots \varphi_{t}\left(x_{n}\right)
$$

${ }^{*}$ Centre Emil Borel, UMS 839 IHP (CNRS/UPMC), Paris. Постоянный адрес: каффедра высшей математики МЭИ (ТУ), Москва 105835, Красноказарменная, 14, Россия. Е-mail: tmph@genesis.mi.ras.ru 
где Е обозначает математическое ожидание.

Предполагается, что процесс $t \mapsto \varphi_{t}$ является решением стохастического уравнения

$$
d \varphi_{t}=-\widehat{K}^{2} \frac{\delta A\left(\varphi_{t}\right)}{\delta \varphi_{t}} d t+\sqrt{2} \widehat{K} d w_{t}, \quad t>0
$$

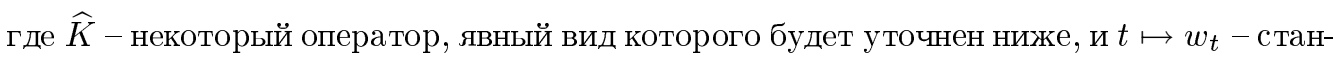
дартный винеровский процесс в $S^{\prime}\left(\mathbb{R}^{d}\right)$, т.е. $\forall s, t>0$ и $\forall f, g \in S\left(\mathbb{R}^{d}\right)$ справедливо равенство

$$
\mathrm{E}\left\langle w_{s}, f\right\rangle\left\langle w_{t}, g\right\rangle=\min \{s, t\}\langle f, g\rangle .
$$

Символ $\langle\cdot, \cdot\rangle$ обозначает форму двойственности между $S^{\prime}\left(\mathbb{R}^{d}\right)$ и $S\left(\mathbb{R}^{d}\right)$, а также скалярное произведение в пространстве $L^{2}\left(\mathbb{R}^{d}\right)$.

Формальное уравнение Фоккера-Планка для уравнения (3) имеет вид

$$
\frac{\partial}{\partial t} P_{t}(\varphi)=\int_{\mathbb{R}^{d}} \widehat{K}^{2} \frac{\partial}{\partial \varphi(x)}\left[\frac{\delta P_{t}(\varphi)}{\delta \varphi(x)}+\frac{\delta A(\varphi)}{\delta \varphi(x)} P_{t}(\varphi)\right] d x,
$$

где $P_{t}$ - “плотность" вероятностного распределения случайного элемента $\varphi_{t}$. Поскольку $\varphi_{t}$ принимает значения в бесконечномерном пространстве, его вероятностное распределение не может иметь плотности. Тем не менее уравнение (4) часто используется в эвристических рассуждениях (см., например, [3]). В частности, уравнение (4) показывает, что

$$
P(\varphi) \sim e^{-A(\varphi)}
$$

- плотность стационарного вероятностного распределения. Кроме того, это стационарное распределение не зависит от выбора $\widehat{K}$.

Оператор $\widehat{K}$ назьвается ядром, а уравнение (3) - модифицированным (kerneled) уравнением Ланжевена. Ссылки на работы, в которых применялось модифицированное уравнение Ланжевена, приведены, например, в [4]. Укажем еще работы [5] и [6]. В них уравнение (3) для модели $\lambda \varphi^{4}$ исследовалось при $d=2$ в конечной области $\Lambda \subset \mathbb{R}^{2}$. Использовалось ядро $\widehat{K}=(-\Delta+1)^{-(1-\varepsilon) / 2}$ при $0<\varepsilon<1$. Тогда $\operatorname{Tr} \widehat{K}^{2}<\infty$ в пополнении $L^{2}(\Lambda)$ по норме $\left\|(-\Delta+1)^{-1 / 2} \cdot\right\|$. Уравнение $(3)$ неявно использовалось также в работах [1] и [2]. В них $d$ - произвольное натуральное число, $\widehat{K}=(-\Delta+1)^{-1 / 2}$ и в качестве гильбертова пространства, в котором $\operatorname{Tr} \widehat{K}^{2}<\infty$, использовалось пополнение $L^{2}\left(\mathbb{R}^{d}\right)$ по норме $\|\widehat{B} \cdot\|$, где $\widehat{B}=\left(1+|x|^{2}\right)^{-b}(-\Delta+1)^{-a}$ при некоторых $b>d / 4$ и $a>(d-2) / 4$

В данной работе мы используем ядро $\widehat{K}=\widehat{H}^{-k}$, где

$$
\widehat{H}=-\Delta+1+|x|^{2}+d \text {. }
$$

Число $k$ выбирается так, чтобы условие $\operatorname{Tr} \widehat{K}^{2}<\infty$ выполнялось в $L^{2}\left(\mathbb{R}^{d}\right)$. Следовательно, $k>d / 2$. Мы используем такое $k$, при котором решение уравнения (3) в каждый 
момент времени принадлежит одному и тому же гильбертову пространству достаточно гладких и быстро убывающих функций. Это пространство описано в разделе 1. Пока обозначим его символом $\Phi$. Важно то, что отображение

$$
\varphi \in \Phi \mapsto \widehat{K}^{2} \frac{\delta A(\varphi)}{\delta \varphi} \in \Phi
$$

определено без каких-либо регуляризаций. Более того мы доказываем в разделе 2, что это отображение удовлетворяет условию Липшица. Следовательно, можно применить стандартные теоремы и доказать, что задача Коши для (3) при любом начальном распределении в пространстве $\Phi$ имеет единственное решение. Оно определено при всех $t>0$ и является марковским процессом.

В разделе 3 мы доказываем, что вероятностные распределения случайных величин $\varphi_{t}$ слабо сходятся при $t \rightarrow \infty$ к некоторой мере на $S^{\prime}\left(\mathbb{R}^{d}\right)$.

В заключении кратко обсуждаются полученные результаты.

\section{1. ПРОСТРАНСТВО $\Phi$}

Для каждого $l \in \mathbb{R}$ определим норму $\|\cdot\|_{l}$ в пространстве $S\left(\mathbb{R}^{d}\right)$ как отображение

$$
f \in S\left(\mathbb{R}^{d}\right) \mapsto\|f\|_{l}:=\left\|\widehat{H}^{l} f\right\|
$$

В частности, $\|\cdot\|_{0}$ - стандартная норма $\|\cdot\|$ в $L^{2}\left(\mathbb{R}^{d}\right)$. Пополнение $S\left(\mathbb{R}^{d}\right)$ относительно нормы $\|\cdot\|_{l}$ обозначим символом $\mathcal{D}\left(\widehat{H}^{l}\right)$. Свойства элементов пространства $\mathcal{D}\left(\widehat{H}^{l}\right)$ характеризуются следуюшей теоремой (ср. [7]).

ТЕОРема 1. Для любого $j=0,1,2, \ldots$ и любого

$$
l>(j+d) / d
$$

имеет место вложение $\mathcal{D}\left(\widehat{H}^{l}\right) \subset C^{(j)}\left(\mathbb{R}^{d}\right)$. Кроме того, существует число $Q_{j}>0$ такое, что $\forall f \in \mathcal{D}\left(\widehat{H}^{l}\right)$ справедливо неравенство

$$
\|f\|_{C^{(j)}} \leq Q_{j}\|f\|_{l}
$$

ДокаЗАТЕльство. Для любых $n_{1}, \ldots, n_{d}=0,1, \ldots$ пусть $\psi_{n_{1}, \ldots, n_{d}}-$ собственная функция оператора $\widehat{H}$, т.е.

$$
\widehat{H} \psi_{n_{1}, \ldots, n_{d}}=\left[2\left(n_{1}+\cdots+n_{d}+d\right)+d\right] \psi_{n_{1}, \ldots, n_{d}}
$$

и

$$
\left\langle\psi_{n_{1}, \ldots, n_{d}}, \psi_{k_{1}, \ldots, k_{d}}\right\rangle=\delta_{n_{1}, k_{1}} \ldots \delta_{n_{d}, k_{d}}
$$


Иначе говоря,

$$
\psi_{n_{1}, \ldots, n_{d}}\left(x_{1}, \ldots, x_{d}\right)=\prod_{k=1}^{d}\left(\pi^{1 / 2} 2^{n_{k}} n_{k} !\right)^{-1 / 2} H_{n_{k}}\left(x_{k}\right) e^{-x_{k}^{2} / 2},
$$

где $H_{n}$ - многочлены Эрмита $n$-й степени в смысле Куранта и Гильберта (см., например, [8]).

Функция $f$ принадлежит $\mathcal{D}\left(\widehat{H}^{l}\right)$ тогда и только тогда, когда

$$
f=\sum_{n_{1}, \ldots, n_{d}=0}^{\infty} c_{n_{1}, \ldots, n_{d}} \psi_{n_{1}, \ldots, n_{d}},
$$

где коэффициенты $c_{n_{1}, \ldots, n_{d}}$ таковы, что

$$
\sum_{n_{1}, \ldots, n_{d}=0}^{\infty}\left[2\left(n_{1}+\cdots+n_{d}\right)+3 d\right]^{2 l}\left|c_{n_{1}, \ldots, n_{d}}\right|^{2}<\infty
$$

Если $l>d / 2$, то ряд (8) сходится равномерно, поскольку из формулы (10.18.49) в [8] следует, что

и, кроме того, справедливо неравенство

$$
\left|\psi_{n_{1}, \ldots, n_{d}}\right|<\left(\frac{2}{\pi}\right)^{d / 4}
$$

$$
\begin{aligned}
\sum_{n_{1}, \ldots, n_{d}=0}^{\infty}\left|c_{n_{1}, \ldots, n_{d}}\right| \leq & \left\{\sum_{n_{1}, \ldots, n_{d}=0}^{\infty}\left[2\left(n_{1}+\cdots+n_{d}\right)+3 d\right]^{-2 l}\right\}^{1 / 2} \times \\
& \times\left\{\sum_{n_{1}, \ldots, n_{d}=0}^{\infty}\left[2\left(n_{1}+\cdots+n_{d}\right)+3 d\right]^{2 l}\left|c_{n_{1}, \ldots, n_{d}}\right|^{2}\right\}^{1 / 2}<\infty
\end{aligned}
$$

Таким образом, если $l>d / 2$, то $\mathcal{D}\left(\widehat{H}^{l}\right) \subset C\left(\mathbb{R}^{d}\right)$ и неравенство (7) выполняется при $j=0$.

Предположим теперь, что теорема справедлива при $j=j_{0}$, где $j_{0}$ - некоторое неотрицательное целое число, и докажем, что она справедлива при $j=j_{0}+1$.

Если $f \in S\left(\mathbb{R}^{d}\right)$, то $\left\|\partial_{i} f\right\|_{C^{(j)}} \leq Q_{j}\left\|\partial_{i} f\right\|_{l_{j}}$. Имеем

$$
\begin{aligned}
\partial_{i} f & =\sum_{n_{1}, \ldots, n_{d}=0}^{\infty} c_{n_{1}, \ldots, n_{d}} \partial_{i} \psi_{n_{1}, \ldots, n_{d}}= \\
& =\sum_{n_{1}, \ldots, n_{d}=0}^{\infty} c_{n_{1}, \ldots, n_{d}}\left[\sqrt{\frac{n_{i}}{2}} \psi_{n_{1}, \ldots, n_{i}-1, \ldots, n_{d}} \sqrt{\frac{n_{i}+1}{2}} \psi_{n_{1}, \ldots, n_{i}+1, \ldots, n_{d}}\right] .
\end{aligned}
$$

Следовательно, $\left\|\partial_{i} f\right\|_{l_{j}} \leq\|f\|_{l_{j}+1 / 2}$ и

$$
\|f\|_{C^{(j+1)}} \leq\|f\|_{C^{(j)}}+\sum_{i=1}^{d}\left\|\partial_{i} f\right\|_{C^{(j)}} \leq Q_{j}\|f\|_{l_{j}}+d\|f\|_{l_{j}+1 / 2} \leq\left(Q_{j}+d\right)\|f\|_{l_{j}+1 / 2} .
$$

Таким образом, если $l \geq l_{j}+1 / 2$, то $\mathcal{D}\left(\widehat{H}^{l}\right) \subset C^{(j+1)}$ и справедливо неравенство $(7)$. Мы получили также, что $Q_{j+1} \geq Q_{j}+d$. 
ЗАмЕчАниЕ. Для моделей с пространственным обрезанием можно использовать оператор $\widehat{H}_{0}$ вместо $\widehat{H}$. Тогда теорема 1 должна быть заменена теоремой Соболева о вложении.

ТЕОРема 2. Для любой функиии $f \in S\left(\mathbb{R}^{d}\right)$ справедливо неравенство

$$
\left\|\widehat{H}_{0} \widehat{H}^{-1} f\right\| \leq\|f\|
$$

$u \forall m=0,1, \ldots, \forall l=0,1 / 2,1,3 / 2, \ldots$ неравенство

$$
\left\|\widehat{H}^{l}|x|^{2 m} f\right\| \leq C_{l, m}\left\|\widehat{H}^{l+m} f\right\| .
$$

ДокАЗАТЕЛЬСТво. Для того чтобы доказать неравенство (9), достаточно заметить, что

$$
\begin{aligned}
\|f\|^{2}-\left\|\widehat{H}_{0} \widehat{H}^{-1} f\right\| & =\left\langle\widehat{H}^{-1} f,\left(\widehat{H}_{0} U+U \widehat{H}_{0}+U^{2}\right) \widehat{H}^{-1} f\right\rangle= \\
& =2\langle\nabla g, U \nabla g\rangle+\left\langle g,\left(U^{2}+2 U-\Delta U\right) g\right\rangle \geq 0,
\end{aligned}
$$

где $U=|x|^{2}+d$ и $g=\widehat{H}^{-1} f$.

Для того чтобы доказать неравенство (10), положим

$$
a_{i}=\frac{1}{\sqrt{2}}\left(x_{i}+\partial_{i}\right) \quad \text { и } \quad a_{i}^{+}=\frac{1}{\sqrt{2}}\left(x_{i}-\partial_{i}\right),
$$

тогда

$$
\widehat{H}=2 \sum_{i=1}^{d} a_{i}^{+} a_{i}+2 d+1
$$

и

$$
|x|^{2}=\sum_{i=1}^{d} a_{i}^{+} a_{i}+\frac{1}{2} \sum_{i=1}^{d}\left[\left(a_{i}^{+}\right)^{2}+a_{i}^{2}\right]+\frac{d}{2} .
$$

Теперь, используя коммутационные соотношения

$$
\left[a_{i_{1}}, a_{i_{2}}^{+}\right]=\delta_{i_{1}, i_{2}}
$$

мы получаем, что

$$
\left\|\widehat{H}^{l}|x|^{2 m} f\right\|^{2} \leq \sum_{\alpha=0}^{l+m} c_{\alpha}\left\|\widehat{H}^{\alpha} f\right\|^{2} \leq\left\|\widehat{H}^{l+m} f\right\|^{2} \sum_{\alpha=0}^{l+m} c_{\alpha} .
$$

Из (7) и (10) следует, что $\forall f \in S\left(\mathbb{R}^{d}\right), \forall m=0,1, \ldots$ и $\forall l=(d+1) / 2,(d+2) / 2, \ldots$ имеем

$$
\left\||x|^{2 m} f\right\|_{C} \leq Q_{0}\left\|\widehat{H}^{l}|x|^{2 m} f\right\| \leq Q_{0} C_{l, m}\left\|\widehat{H}^{l+m} f\right\|
$$


Таким образом, $\forall f \in \mathcal{D}\left(\widehat{H}^{l+m}\right), \forall m=0,1, \ldots$ и $\forall l=(d+1) / 2,(d+2) / 2, \ldots$ справедливо неравенство

$$
\left\||x|^{2 m} f\right\|_{C} \leq G_{l, m}\|f\|_{l+m}
$$

при некотором $G_{l, m}>0$.

Теперь определим пространство $\Phi$, в котором содержатся выборочные траектории процесса $t \mapsto \varphi_{t}$

Поскольку $\mathrm{E}\left\langle w_{t}, f\right\rangle^{2}=t\|f\|^{2}$, то $w_{t}$ почти наверное лежит в $\mathcal{D}\left(\widehat{H}^{-k_{0}}\right)$ при любом $k_{0}>d / 2$. Следовательно

$$
\widehat{K} w_{t}=\widehat{H}^{-k} w_{t} \in \mathcal{D}\left(\widehat{H}^{k-k_{0}}\right)
$$

Выберем некоторые $k_{0}>d / 2$ и $k>k_{0}$ такие, что

$$
k-k_{0} \geq(d+3) / 2
$$

Затем положим

$$
\Phi=\mathcal{D}\left(\widehat{H}^{k-k_{0}}\right)
$$

По теореме 1

$$
\Phi \subset C^{(2)}\left(\mathbb{R}^{d}\right)
$$

Используя $(11)$ с $m=1$ и $l=(d+1) / 2$, мы получаем, что

$$
f \in \Phi \Rightarrow\left\||x|^{2} f\right\|_{C} \leq G\|f\|_{\Phi}
$$

Итак, каждый элемент пространства $\Phi$ есть функция $\mathbb{R}^{d} \mapsto \mathbb{R}$, которая стремится к нулю при $|x| \rightarrow \infty$ и имеет ограниченные непрерьвные производные первого и второго порядков.

Дополнительно к (12) мы предполагаем, что число $k$ таково, что выполняются следующие два условия:

$$
\Phi \subset \mathcal{D}\left(\widehat{H}_{0}^{1 / 2}\right)
$$

$$
\forall p \in\left[2, \infty\left[\quad \Phi \subset L^{p}\left(\mathbb{R}^{d}\right),\right.\right.
$$

причем

$$
\forall \varphi \in \Phi \quad\|\varphi\|_{L^{p}\left(\mathbb{R}^{d}\right)} \leq O(1)\|\varphi\|_{\Phi}
$$


ЗАМЕЧАНИЯ.

1. Поскольку $\forall \varphi \in S\left(\mathbb{R}^{d}\right)$ справедливо неравенство

$$
\left\|\widehat{H}_{0}^{1 / 2} \varphi\right\| \leq\left\|\widehat{H}^{1 / 2} \varphi\right\|,
$$

условие (15) выполняется при $k-k_{0}>1 / 2$, т.е. заведомо при всех $k$ и $k_{0}$, для которых справедливо неравенство (12).

2. Вложение (16) справедливо, поскольку $\Phi \subset L^{2}\left(\mathbb{R}^{d}\right)$ и $\Phi \subset C\left(\mathbb{R}^{d}\right)$.

3. Неравенство (17) действительно выполняется при достаточно большом числе $k$. В этом можно убедиться, заметив, что справедливо неравенство

$$
\begin{aligned}
\|\varphi\|_{L^{p}\left(\mathbb{R}^{d}\right)}^{p} & \leq \sup _{x \in \mathbb{R}^{d}}\left(1+|x|^{2}\right)^{(d+1) / 2}|\varphi(x)|^{p} \int_{\mathbb{R}^{d}} \frac{d z}{\left(1+|z|^{2}\right)^{(d+1) / 2}}= \\
& =\left[\sup _{x \in \mathbb{R}^{d}}\left(1+|x|^{2}\right)^{(d+1) /(2 p)}|\varphi(x)|\right]^{p} \int_{\mathbb{R}^{d}} \frac{d z}{\left(1+|z|^{2}\right)^{(d+1) / 2}} .
\end{aligned}
$$

Поэтому, если $m$ - натуральное число, большее чем $(d+1) /(2 p)$, то в силу $(11)$ неравенство (17) выполняется при

$$
k-k_{0} \geq m+(d+1) / 2 .
$$

\section{2. ПРОЦЕСС $t \mapsto \varphi_{t}$}

В этом разделе мы исследуем уравнение (3).

Теорема 3. Отображение (5) принимает значения в пространстве $\Phi$ и $\forall \varphi$, $\psi \in \Phi$ справедливо неравенство

$$
\left\|\widehat{K}^{2} \frac{\delta A(\varphi)}{\delta \varphi}-\widehat{K}^{2} \frac{\delta A(\psi)}{\delta \psi}\right\|_{2 k-1} \leq(C+1)\|\varphi-\psi\|
$$

ДокАЗАТЕЛЬСтво. Для любого $q \in \mathcal{D}\left(\widehat{H}^{-2 k+1}\right)$ и любого $\varphi \in \Phi$ справедливы неравенства

$$
\left|\left\langle\widehat{K}^{2} \frac{\delta A(\varphi)}{\delta \varphi}, q\right\rangle\right| \leq\left|\left\langle v^{\prime}(\varphi), \widehat{H}^{-2 k} q\right\rangle\right|+\left|\left\langle\varphi, \widehat{H}_{0} \widehat{H}^{-2 k} q\right\rangle\right| \leq(C+1)\|\varphi\|\left\|\widehat{H}^{-2 k+1} q\right\| .
$$

Следовательно,

И

$$
\left\|\widehat{K}^{2} \frac{\delta A(\varphi)}{\delta \varphi}\right\|_{2 k-1} \leq(C+1)\|\varphi\|
$$

$$
\left\|\widehat{K}^{2} \frac{\delta A(\varphi)}{\delta \varphi}\right\|_{\Phi} \leq(C+1)\|\varphi\|_{\Phi}
$$

поскольку

$$
\|\cdot\| \leq\|\cdot\|_{\Phi}=\|\cdot\|_{k-k_{0}} \leq\|\cdot\|_{2 k-1} .
$$

Таким образом, отображение (5) принимает значения в $\Phi$. Аналогично можно доказать неравенство (18). 
ЗАмечАниЕ. На самом деле, мы доказали, что отображение (5) действует из $L^{2}\left(\mathbb{R}^{d}\right)$ в $\mathcal{D}\left(\widehat{H}^{2 k-1}\right) \subset \Phi$.

Используя (18) и (19), получаем, что отображение (5) удовлетворяет следуюшему условию Липшица:

$$
\forall \varphi, \psi \in \Phi \quad\left\|\widehat{K}^{2} \frac{\delta A(\varphi)}{\delta \varphi}-\widehat{K}^{2} \frac{\delta A(\psi)}{\delta \psi}\right\|_{\Phi} \leq(C+1)\|\varphi-\psi\|_{\Phi}
$$

Следовательно, уравнение (3) является стохастическим уравнением типа Ито в гильбертовом пространстве $\Phi$ с липшицевым коэффициентом сноса $\widehat{K}^{2} \delta A\left(\varphi_{t}\right) / \delta \varphi_{t}$. В силу стандартных теорем (см., например, [9, гл. VII.2.1]) при любом вероятностном распределении начального значения $\varphi_{0}$ в $\Phi$ сушествует единственное решение $t \mapsto \varphi_{t}$ уравнения (3). Это решение определено при всех $t \geq 0$ и является однородным по времени марковским процессом.

\section{3. АСИМПТОТИЧЕСКОЕ ПОВЕДЕНИЕ СЛУЧАЙНЫХ ЭЛЕМЕНТОВ $\varphi_{t}$}

Здесь мы докажем, что вероятностные распределения случайных элементов $\varphi_{t}(t \geq 0)$ слабо сходятся к некоторой вероятностной мере на $S^{\prime}\left(\mathbb{R}^{d}\right)$. Начнем с того, что установим одно соотношение для $\mathrm{E}\left\|\widehat{K} \widehat{H}_{0} \varphi_{t}\right\|^{2}$.

ТЕорема 4. Для любого $\varphi_{0}$, для которого $\mathrm{E}\left\|\widehat{H}_{0}^{1 / 2} \varphi_{0}\right\|<\infty$, существует последовательность $t_{1}<t_{2}<\cdots<t_{n}<\cdots$ такая, что $\lim _{n \rightarrow \infty} t_{n}=\infty u$

$$
\sup _{n} \mathrm{E}\left\|\widehat{K} \widehat{H}_{0} \varphi_{t_{n}}\right\|^{2}<\infty .
$$

ДоКАЗАТЕЛЬСТво. Используя формулу Ито для $\left\|\widehat{H}_{0}^{1 / 2} \varphi_{t}\right\|^{2}$, мы получаем, что

$$
\begin{aligned}
\mathrm{E}\left\|\widehat{H}_{0}^{1 / 2} \varphi_{t}\right\|^{2}= & \mathrm{E}\left\|\widehat{H}_{0}^{1 / 2} \varphi_{0}\right\|^{2}+2 t \operatorname{Tr} \widehat{K} \widehat{H}_{0} \widehat{K}- \\
& -2 \int_{0}^{t} \mathrm{E}\left[\left\|\widehat{K} \widehat{H}_{0} \varphi_{s}\right\|^{2}+\left\langle\widehat{K} \widehat{H}_{0} \varphi_{s}, \widehat{K} v^{\prime}\left(\varphi_{s}\right)\right\rangle\right] d s .
\end{aligned}
$$

Пусть

$$
\begin{aligned}
a & =\mathrm{E}\left\|\widehat{H}_{0}^{1 / 2} \varphi_{0}\right\|^{2}, \\
b & =2 \operatorname{Tr} \widehat{K} \widehat{H}_{0} \widehat{K}, \\
c & =\sup _{\varphi \in \Phi}\left\|\widehat{K} v^{\prime}(\varphi)\right\|
\end{aligned}
$$

и

$$
U(t)=\int_{0}^{t} \mathrm{E}\left\|\widehat{K} \widehat{H}_{0} \varphi_{s}\right\|^{2} d s
$$


тогда

$$
0 \leq a+b t+2 c \sqrt{t U(t)}-2 U(t)
$$

Следовательно,

$$
\int_{0}^{t} \mathrm{E}\left\|\widehat{K} \widehat{H}_{0} \varphi_{s}\right\|^{2} d s \leq a+\left(a+b+c^{2}\right) \frac{t}{2}
$$

Теперь утверждение теоремы очевидно.

Используя неравенство Чебышева, мы выводим из (21), что $\forall n \in \mathbb{N}$ имеем

$$
\operatorname{Prob}\left\{\left\|\widehat{K} \widehat{H}_{0} \varphi_{t_{n}}\right\|>r\right\} \leq \frac{D}{r^{2}}
$$

где $D$ - левая часть неравенства $(21)$.

Обозначим символом $P_{n}$ вероятностное распределение $\varphi_{t_{n}}$, а символом $\mu_{n}-$ расширение $P_{n}$ на пополнение $\mathcal{D}\left(\widehat{H}^{-\varepsilon} \widehat{K} \widehat{H}_{0}\right)$ пространства $\mathcal{D}\left(\widehat{K} \widehat{H}_{0}\right)$ по норме $\| \widehat{H}^{-\varepsilon} \widehat{K} \widehat{H}_{0}$. $\|$ при некотором $\varepsilon>0$. Естественное вложение $\mathcal{D}\left(\widehat{K} \widehat{H}_{0}\right) \subset \mathcal{D}\left(\widehat{H}^{-\varepsilon} \widehat{K} \widehat{H}_{0}\right)$ компактно. Следовательно, семейство мер $\mu_{n} \quad(n \in \mathbb{N})$ на $\mathcal{D}\left(\widehat{H}^{-\varepsilon} \widehat{K} \widehat{H}_{0}\right)$ слабо предкомпактно. Таким образом, сушествуют последовательность натуральных чисел $n_{1}<n_{2}<\cdots$ и вероятностная мера $\mu$ на $\mathcal{D}\left(\widehat{H}^{-\varepsilon} \widehat{K} \widehat{H}_{0}\right)$ такие, что последовательность $\mu_{n_{1}}, \mu_{n_{2}}, \ldots$ слабо сходится к $\mu$.

\section{ЗАКЛЮЧЕНИЕ}

Использование модифицированного уравнения Ланжевена с подходящим ядром $\widehat{K}$ позволило нам обойтись без каких-либо промежуточных регуляризаций. Заметим, что регуляризации необходимы тогда, когда требуется определить функционал действия на пространстве обобщенных функций и затем строить меру Нельсона как слабьй предел мep

$$
e^{-\int \mathcal{L}_{\mathrm{int}}^{\mathrm{rag}}(\varphi(x)) d x} d \mu_{\mathrm{free}}(\varphi)
$$

при снятии регуляризации. В данной статье функционал действия не продолжался с того пространства гладких функций, на котором он определен в классической теории. Вместо этого использовалось продолжение вероятностных распределений случайных элементов $\varphi_{t}(t \geq 0)$. Эти распределения изначально заданы на пространстве $\Phi$. Мы продолжили их с $\Phi$ на $S^{\prime}\left(\mathbb{R}^{d}\right)$ и показали, что продолженные вероятностные распределения слабо сходятся к некоторой вероятностной мере на $S^{\prime}\left(\mathbb{R}^{d}\right)$. Заметим, что продолжение мер в отличие от продолжения функционалов обычно не вызывает никаких затруднений.

Предположим, что $\forall t>0$ вероятностное распределение случайного элемента $\varphi_{t}$ может быть представлено в виде

$$
\varrho\left(\varphi_{t}\right) d \mu_{\text {free }}\left(\varphi_{t}\right)
$$


Если это действительно так, то сравнивая (22) и (23), можно узнать, какой регуляризации эквивалентно использование стохастического квантования с помошью модифицированного уравнения Ланжевена. Понятно, что регуляризованные функционалы действия зависят только от одного параметра $t$, а не от нескольких параметров типа размеров пространственного и импульсного обрезаний. Этим стохастическое квантование отличается от других способов построения квантово-полевых моделей.

Коэффициент сноса $\widehat{K}^{2} \delta A / \delta \varphi$ для полиномиальных моделей типа $\lambda \varphi^{4}$ удовлетворяет условию Липшица лишь локально (ср. с теоремой 3 ). Тем не менее можно доказать, что для модели $\lambda \varphi^{4}$ в пространстве-времени произвольной размерности уравнение (3) имеет решения, определенные при всех $t>0$. Мы надеемся опубликовать результаты, относящиеся к модели $\lambda \varphi^{4}$, в отдельной статье.

Эта работа выполнена во время пребывания автора в институте им. А. Пуанкаре (Франция) в рамках организованного центром Э. Бореля семестрового цикла лекций и семинаров, посвященных функциональному интегрированию. Автор благодарен организаторам цикла Ж.-М. Бисмю, С. де Витт-Моретт и П. Картье за предоставленную возможность принять участие в работе цикла. Автор благодарен также профессорам Д. Бриджесу и С. де Витт-Моретт за весьма полезные замечания и О. В. Зиминой за постоянную помошь и заботу.

Работа выполнена при финансовой поддержке РФФИ (проект № 96-01-00726).

\section{Список литературы}

[1] А.И. Кириллов. ТМФ. 1994. Т. 98. С. 12-28.

[2] А. И. Кириллов. ТМФ. 1995. Т. 105. С. 179-197.

[3] M. Namiki. Stochastic Quantization. Berlin: Springer, 1992.

[4] О.И. Завьялов, М. Каненага, А.И. Кириллов, В. Ю. Мамакин, М. Намики, И. Охба, Е. В. Поляченко. ТМФ. 1996. Т. 109. С. 175-186.

[5] G. Jona-Lasinio, S. K. Mitter. Commun. Math. Phys. 1985. V. 101. P. 409-436.

[6] B.S. Borkar, R. T. Chari, S. K. Mitter. J. Funct. Anal. 1988. V. 81. P. 184-206.

[7] B. Simon. J. Math. Phys. 1971. V. 12. P. 140-148.

[8] Г. Бейтмен, А. Эрдейи. Высшие трансцендентные функции. Т. 2. М.: Наука, 1974.

[9] Ю. Л. Далецкий, С. В. Фомин. Меры и дифференциальные уравнения в бесконечномерном пространстве. М.: Наука, 1983. 